\title{
Risk factors for possible REM sleep behavior disorder
}

\author{
A CLSA population-based cohort study
}

Chun Yao, MSc, Seyed-Mohammad Fereshtehnejad, MD, MPH, PhD, Mark R. Keezer, MDCM, PhD, Christina Wolfson, PhD, Amélie Pelletier, PhD, and Ronald B. Postuma, MD, MSc

Neurology ${ }^{\circledR}$ 2019;92:e475-e485. doi:10.1212/WNL.0000000000006849

\section{Abstract}

\section{Objective}

To assess sociodemographic, socioeconomic, and clinical correlates of idiopathic REM sleep behavior disorder (RBD) in a 30,097-person national cohort.

\section{Methods}

Participants 45 to 85 years of age in Canada were collected as part of the Canadian Longitudinal Study on Aging. Possible RBD (pRBD) was screened with the REM Sleep Behavior Disorder Single-Question Screen, a questionnaire with 94\% specificity and $87 \%$ sensitivity. To improve diagnostic accuracy, those screening positive for apnea or non-REM parasomnia (young-onset $\mathrm{pRBD}$ ) and those self-reporting dementia or Parkinson disease were excluded. A series of sociodemographic, lifestyle, and mental health variables were analyzed cross-sectionally. Potential correlates were assessed via multivariable logistic regression.

\section{Results}

Of 30,097 participants, 958 (3.2\%) were identified as having pRBD. Male sex (odds ratio [OR] 2.09, 95\% confidence interval [CI] 1.78-2.44) and lower education (OR 0.95, 95\% CI 0.92-0.98) were associated with pRBD. Participants with pRBD had smoked more (pack-years OR 1.01, 95\% CI 1.00-1.01) and were more likely to be moderate to heavy drinkers (OR 1.25, 95\% CI 1.04-1.51). There was a strong association between pRBD and self-reported antidepressant treatment for depression (OR 2.77, 95\% CI 2.23-3.45), psychological distress (OR 1.61, 95\% CI 1.44-1.80), mental illness (OR 2.09, 95\% CI 1.75-2.49), and posttraumatic stress disorder (OR 2.68, 95\% CI 1.97-3.65).

\section{Conclusions}

Our study replicated previous reported associations between pRBD and smoking, low education, and male sex and found previously unreported links with alcohol use and psychological distress. Risk factors for $\mathrm{pRBD}$ differ from those previously defined for neurodegenerative synucleinopathies.

\author{
Correspondence \\ Dr. Postuma \\ ron.postuma@mcgill.ca
}

From the Integrated Program in Neuroscience (C.Y.), Department of Neurology and Neurosurgery (S.-M.F., R.B.P.), Department of Epidemiology and Biostatistics and Occupational Health (C.W.), and Department of Medicine (C.W., A.P.), McGill University; Centre de Recherche du Centre hospitalier de I'Université de Montréal (M.R.K.); and Research Institute of the McGill University Health Centre (C.W., A.P.), Montreal, Quebec, Canada.

Go to Neurology.org/N for full disclosures. Funding information and disclosures deemed relevant by the authors, if any, are provided at the end of the article. 


\section{Glossary}

CI = confidence interval; CLSA = Canadian Longitudinal Study on Aging; DLB = dementia with Lewy bodies; NREM = nonREM; OR = odds ratio; PD = Parkinson disease; PLMS = periodic leg movements in sleep; pRBD = possible REM sleep behavior disorder; PSG = polysomnogram; PTSD = posttraumatic stress disorder; RBD = REM sleep behavior disorder; RBD1Q = REM Sleep Behavior Disorder Single-Question Screen; RLS = restless leg syndrome.

REM sleep behavior disorder (RBD) is a disorder in which loss of the normal REM sleep paralysis allows patients to act out their dreams. The prevalence of polysomnogram (PSG)confirmed $\mathrm{RBD}$ is $\approx 1 \%$ in those $>50$ years of age. ${ }^{1-3} \mathrm{RBD}$ is the strongest known prodromal symptom of neurodegenerative synucleinopathy; $\approx 80 \%$ of patients with PSG-confirmed RBD will develop Parkinson disease (PD), dementia with Lewy bodies (DLB), or multiple system atrophy. Although definitive diagnosis requires PSG, given the expense and limited number of specialists, several questionnaires have been developed to screen for RBD. ${ }^{4-6}$ One of these questionnaires, the REM Sleep Behavior Disorder SingleQuestion Screen (RBD1Q), is a single-question screen that was found to have $94 \%$ specificity and $87 \%$ sensitivity in a large PSG-confirmed cohort study. ${ }^{7}$

Risk factors for RBD are relatively understudied. Some studies have suggested that risk factors associated with PD or dementia (e.g., head trauma) are also present in RBD. However, some risk factors may differ, most notably smoking and caffeine use. ${ }^{8,9}$

We used baseline data, collected between 2012 and 2015, from the Canadian Longitudinal Study on Aging (CLSA) comprehensive sample of 30,097 participants. The CLSA included the $\mathrm{RBD} 1 \mathrm{Q}$ and additional questions to help rule out $\mathrm{RBD}$ mimics. In this study, we assessed sociodemographic, socioeconomic, and clinical correlates of possible RBD (pRBD).

\section{Methods}

\section{CLSA cohort}

The CLSA is a prospective, national, population-based cohort that recruited 51,338 participants 45 to 85 years of age randomly sampled from 10 Canadian provinces and stratified by age. ${ }^{10}$ Written informed consent was obtained from all participants (or guardians of participants) in the study. CLSA participants provide a core information set on demographics, lifestyle and behavior, social, physical, clinical, psychological, economic, and health status measures, including screens for selected neurologic diseases. Of the entire cohort, 30,097 are included in a comprehensive cohort, recruited from 2012 to 2015, in which participants also have in-home face-to-face interviews (including a sleep questionnaire module that screens for sleep onset and maintenance insomnia, excessive somnolence, restless leg syndrome [RLS], and RBD), physical assessments, and biospecimen sampling; this is the sample for the current study. ${ }^{10}$ Data access for the use of this study was reviewed and granted by the Data and Sample Access Committee.

\section{RBD case definition}

In the comprehensive cohort, RBD was screened as a "yes" response to the single-question RBD1Q: "Have you ever been told, or suspected yourself, that you seem to 'act out your dreams' while asleep (e.g., punching, flailing your arms in the air, making running movements, etc.) ? ${ }^{7}$ Specificity has been estimated as $87 \%$; false positives can occur if the RBD1Q detects other sleep disorders, especially non-REM (NREM) sleep disorders and obstructive sleep apnea. Thus, for the primary analysis (figure), to reduce false positives, we excluded screen positives with the following:

1. Onset before 20 years of age. NREM parasomnia is generally a childhood-onset disorder, ${ }^{11,12}$ whereas synucleinopathymediated RBD generally starts after age $40 .^{13}$

2. Positive apnea screen defined as a "yes" response to either of the 2 symptom items from the STOP-BANG questionnaire (i.e., snoring loud enough to be heard in the next room and/or stopping breathing while sleeping). ${ }^{14}$

3. A diagnosis of dementia/Alzheimer disease or parkinsonism/PD (i.e., not idiopathic RBD).

In the risk factor assessment, these participants were excluded from both the RBD screen-positive and RBD screen-negative groups (i.e., the control group) to prevent bias related to risk factors for the excluded conditions (e.g., if apnea is removed from the RBD group but not the control group, apnea risk factors would be imbalanced between groups). Note that the use of the term risk factor here does not imply temporality or causality because this is a cross-sectional study.

\section{Sociodemographic and lifestyle variables}

Educational levels were categorized in the CLSA as middle school and under, secondary school, bachelor degree and other diploma, and above. For the purposes of this analysis, total years of education were imputed from the estimated years in the education categories and from information provided directly in follow-up questions $(0-8$ years imputed as the average $=4,9-10$ taken from follow-up questions, secondary school imputed as 12 , postsecondary imputed according to average length of degree for each diploma type). Marital status was categorized into single/never married, married/common-law, widowed, and divorced/separated. Annual personal income was grouped into 4 levels: $<\$ 20,000, \$ 20,000$ to $\$ 49,000, \$ 50,000$ to $\$ 99,000$, and $\geq \$ 100,000$. For ethnicity, participants were classified as white 


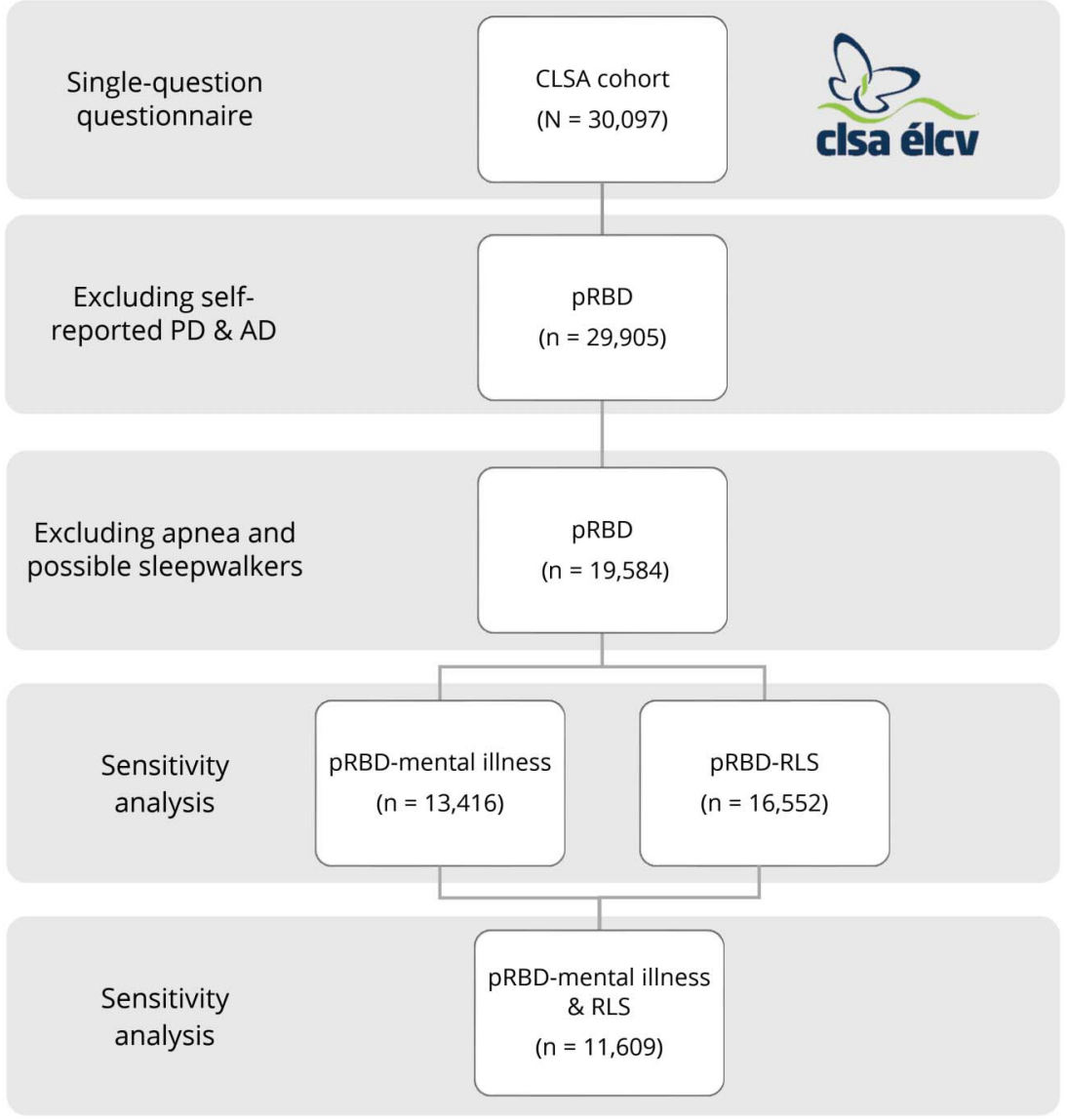

AD = Alzheimer disease $:$ CLSA = Canadian Longitudinal Study on Aging; PD = Parkinson disease; $p$ RBD = possible REM sleep behavior disorder; RLS = restless leg syndrome. or nonwhite (95.6\% were white, so we did not have sufficient power to subdivide nonwhite ethnicities).

For lifestyle variables, we analyzed the average weekly walking hours during leisure time and the annual frequency of participating in social activities. Smoking status was categorized into 3 groups: never smoker (combining occasional smokers and nonsmokers), past smoker, and current smoker. We calculated pack-years of smoking as packs per day times smoking years. Weekly alcohol consumption was based on self-report using standard alcoholic beverage amounts (14 g ethanol). Binge drinking frequency was defined as $>5$ drinks per sitting for men ( $>4$ for women). Moderate to heavy drinking was defined as drinking $>7$ drinks per week for women and 14 for men. Overall satisfaction level of life and self-rated social standing in the community were rated with the 10-point MacArthur scales. ${ }^{15}$ Self-rated health profiles (healthy aging, mental health, and physical health) were assigned from 1 (poor) to 5 (excellent). Use of antidepressants and mental illness were assessed via self-report.

\section{Statistical analysis of risk factors}

Prevalence odds ratios (ORs) were estimated first from logistic regression adjusted for age and sex (unweighted to the general Canadian population) with $\mathrm{pRBD}$ as the dependent variable. We then reassessed all ORs in a more complete multivariable regression model that included age (continuous), sex (categorical), currently married or widowed (categorical), imputed years of education (continuous), income (ordinal), retirement (categorical), heavy drinking (categorical), daily smoking (categorical), having served in the military (categorical), and mental illness and/or use of antidepressant (categorical). To avoid repetition in analyses, a variable structure similar to the regression model was used to replace the corresponding core variables (e.g., in analyzing the relationship between each age group and RBD, the ordinal variable age group would replace age as a continuous variable in the regression model). Statistical analyses were performed by PASW Statistics 18 (Chicago, IL). We omitted any responses labeled as uncertain or "refused to answer" in all analyses.

\section{Sensitivity analyses}

In addition to the primary analysis, we performed 3 sensitivity analyses (figure e-1 available from dryad, doi.org/10.5061/ dryad.0k27q21).

1. Including all RBD screen positives, i.e., including early onset and with positive apnea screen (still excluding dementia and parkinsonism). 
Table 1 Sociodemographic variables $(n=19,584)$

\begin{tabular}{|c|c|c|c|c|c|}
\hline & & $\begin{array}{l}\text { pRBD+ } \\
(\mathrm{n}=958)\end{array}$ & $\begin{array}{l}\text { pRBD- } \\
(n=18,626)\end{array}$ & $\begin{array}{l}\text { OR }(95 \% \mathrm{Cl}) \text {, age/sex } \\
\text { adjusted only }\end{array}$ & $\begin{array}{l}\text { OR }(95 \% \mathrm{Cl}) \text {, } \\
\text { multivariate model }\end{array}$ \\
\hline \multirow[t]{5}{*}{ Age } & Mean $\pm S D, y$ & $63.0 \pm 10.5$ & $63.5 \pm 10.5$ & $0.994(0.987-1.000)$ & $0.989(0.980-0.998)$ \\
\hline & 45-54 y, n (\%) & $251(26.2)$ & $4,592(24.7)$ & - & - \\
\hline & $55-64$ y, n (\%) & $303(31.6)$ & $5,721(30.7)$ & $0.97(0.82-1.15)$ & $0.85(0.69-1.04)$ \\
\hline & $65-74$ y, n (\%) & $214(22.3)$ & $4,624(24.8)$ & $0.85(0.70-1.02)$ & $0.72(0.56-0.94)$ \\
\hline & $75-85$ y, n (\%) & $190(19.8)$ & 3,689 (19.8) & $0.94(0.78-1.14)$ & $0.79(0.59-1.04)$ \\
\hline Sex, n (\%) & Male & $564(58.9)$ & $7,874(42.3)$ & $1.97(1.72-2.25)$ & $2.09(1.78-2.44)$ \\
\hline Ethnicity, n (\%) & White & 909 (99.2) & $17,794(97.1)$ & $0.94(0.70-1.29)$ & $0.87(0.60-1.26)$ \\
\hline \multirow[t]{4}{*}{ Marital status, n (\%) } & Single/never married & $52(6.1)$ & $1,492(8.7)$ & - & - \\
\hline & Married/common-law & $643(75.1)$ & $11,335(65.8)$ & $1.59(1.19-2.12)$ & $1.79(1.32-2.42)$ \\
\hline & Widowed & $82(9.6)$ & $1906(11.4)$ & $1.47(1.02-2.12)$ & $1.54(1.05-2.27)$ \\
\hline & Divorced/separated & $79(7.2)$ & $2,455(14.2)$ & $1.01(0.71-1.44)$ & $0.98(0.68-1.42)$ \\
\hline \multirow[t]{4}{*}{ Education level, n (\%) } & BS degree and above & $389(40.6)$ & $8,572(46.1)$ & - & - \\
\hline & Secondary school & $494(51.8)$ & $8,982(48.3)$ & $1.77(1.36-2.31)$ & $1.61(1.18-2.20)$ \\
\hline & Primary/middle school & $73(7.6)$ & $1,041(5.6)$ & $1.32(1.15-1.52)$ & $1.25(1.07-1.47)$ \\
\hline & Imputed years of education & $13.2 \pm 2.8$ & $13.6 \pm 2.6$ & $0.94(0.92-0.96)$ & $0.96(0.93-0.98)$ \\
\hline \multirow[t]{4}{*}{ Annual income level, n (\%) } & $<\$ 20,000$ & $159(17.3)$ & 2,799 (15.9) & - & - \\
\hline & $\$ 20,000-\$ 49,000$ & $336(36.6)$ & $6,680(37.9)$ & $0.81(0.66-0.98)$ & $0.97(0.77-1.21)$ \\
\hline & $\$ 50,000-\$ 99,000$ & $308(33.6)$ & $5,851(33.2)$ & $0.73(0.60-0.90)$ & $0.99(0.78-1.25)$ \\
\hline & $>\$ 100,000$ & $114(12.4)$ & $2,302(13.1)$ & $0.60(0.47-0.78)$ & $0.90(0.67-1.22)$ \\
\hline \multirow[t]{4}{*}{ Employment status } & Employed, n (\%) & $400(41.9)$ & $7,885(42.5)$ & - & - \\
\hline & Retired, n (\%) & $554(58.1)$ & $10,669(57.5)$ & $1.22(1.02-1.46)$ & $1.12(0.92-1.36)$ \\
\hline & Retirement age, y & $57.5 \pm 6.8$ & $58.6 \pm 6.6$ & $0.98(0.96-0.99)$ & $0.983(0.967-0.999)$ \\
\hline & Health-related retirement, n (\%) & $164(28.9)$ & $2,393(22.0)$ & $1.46(1.21-1.77)$ & $1.30(1.05-1.62)$ \\
\hline \multirow[t]{2}{*}{ Military service } & Yes, n (\%) & $116(12.1)$ & $1,515(8.1)$ & $1.25(0.99-1.57)$ & $1.22(0.97-1.54)$ \\
\hline & Years of service & $12.11 \pm 12.47$ & $8.93 \pm 11.07$ & $1.03(1.01-1.05)$ & $1.03(1.01,1.05)$ \\
\hline
\end{tabular}

Abbreviations: $\mathrm{Cl}$ = confidence interval; $\mathrm{OR}$ = odds ratio; $\mathrm{PRBD}=$ possible REM sleep behavior disorder.

2. Excluding those with mental illness (to assess possible role of posttraumatic stress disorder [PTSD] and antidepressant-caused RBD); in this analysis, those who screened positive on the Centre for Epidemiologic Studies Depression Scale Revised ${ }^{16}$ or on the Primary Care PTSD Screen, ${ }^{17}$ who scored $>24$ on the Kessler Psychological Distress Scale, ${ }^{18}$ or who self-reported a physician diagnosis of mood disorder, anxiety disorder, or depressive disorder were excluded.

3. Excluding those screening positive for RLS (a possible mimic because of associated periodic leg movements of sleep) with the use of sleep module questions adapted from the Johns Hopkins telephone interview for $\operatorname{RLS}^{19,20}$

\section{Data availability}

Because of the confidentiality and security concerns, only applicants with a CLSA-approved project and the members of the project teams with a signature on Schedule F of the CLSA Access Agreement form are allowed to have direct access to the raw data.

\section{Results}

\section{Characteristics of the cohort}

Of the 30,097 included, 14,777 were male and 15,320 were female; $64.7 \%$ were either married or in a common-law relationship. A total of 3,271 screened positive for pRBD. Of 
Table 2 Lifestyle and life satisfaction $(n=19,584)$

\begin{tabular}{|c|c|c|c|c|c|}
\hline & & $\begin{array}{l}\text { pRBD+ } \\
(n=958)\end{array}$ & $\begin{array}{l}\text { PRBD- } \\
(n=18,626)\end{array}$ & $\begin{array}{l}\text { OR }(95 \% \mathrm{CI}) \text {, age/sex } \\
\text { adjusted only }\end{array}$ & $\begin{array}{l}\text { OR }(95 \% \mathrm{CI}) \text {, } \\
\text { multivariate model }\end{array}$ \\
\hline \multirow[t]{4}{*}{ Walk } & Hours/week (mean \pm SD) & $4.3 \pm 4.5$ & $4.6 \pm 0.9$ & $0.98(0.97-1.00)$ & $0.99(0.97-1.00)$ \\
\hline & $0 \mathrm{~h} / \mathrm{wk}, \mathrm{n}(\%)$ & $137(15.8)$ & $2,497(14.3)$ & - & - \\
\hline & $<7$ h/wk, n (\%) & $548(63.2)$ & $10,832(61.9)$ & $0.91(0.75-1.10)$ & $0.97(0.79-1.19)$ \\
\hline & $\geq 7$ h/wk, n (\%) & $182(21.0)$ & $4,161(23.8)$ & $0.78(0.62-0.98)$ & $0.83(0.65-1.05)$ \\
\hline \multirow{2}{*}{$\begin{array}{l}\text { Frequency of social } \\
\text { activity per year), } \\
\text { mean } \pm \text { SD }\end{array}$} & Social sport & $54.3 \pm 93.7$ & $63.2 \pm 16.1$ & $0.999(0.998-1.000)$ & $1.000(0.999-1.000)$ \\
\hline & Social activity & $25.0 \pm 26.0$ & $26.6 \pm 4.9$ & $0.998(0.996-1.001)$ & $0.999(0.996-1.002)$ \\
\hline \multirow[t]{6}{*}{ Drinking } & No drink last year, n (\%) & $106(11.4)$ & $3,116(11.7)$ & - & - \\
\hline & Occasional drinker, n (\%) & $97(10.4)$ & $2,325(12.8)$ & $1.06(0.86-1.31)$ & $1.17(0.92-1.49)$ \\
\hline & Regular drinker, n (\%) & $730(78.2)$ & $13,701(75.5)$ & $0.83(0.63-1.10)$ & $0.96(0.69-1.31)$ \\
\hline & Alcohol amount, 100 g/wk & $1.0 \pm 2.1$ & $0.7 \pm 1.0$ & $1.10(1.03-1.17)$ & $1.09(1.02-1.16)$ \\
\hline & Binge drinking, frequency/wk & $1.3 \pm 4.6$ & $1.0 \pm 3.7$ & $1.013(0.996-1.030)$ & $1.01(0.99-1.03)$ \\
\hline & Moderate to heavy drinker, n (\%) & $181(18.9)$ & $2,792(14.3)$ & $1.38(1.17-1.63)$ & $1.25(1.04-1.51)$ \\
\hline \multirow[t]{5}{*}{ Smoking } & Cigarette pack-years & $8.4 \pm 14.7$ & $6.1 \pm 12.2$ & $1.008(1.003-1.013)$ & $1.01(1.00-1.01)$ \\
\hline & Never daily smoker, n (\%) & $462(48.9)$ & $10,269(56.2)$ & - & - \\
\hline & $\begin{array}{l}\text { Ever smoking (reference = never } \\
\text { daily smoker), } \mathrm{n}(\%)\end{array}$ & $493(51.6)$ & $8,235(44.5)$ & $1.28(1.11-1.48)$ & $1.14(0.99-1.33)$ \\
\hline & Past daily smoker, n (\%) & $408(42.7)$ & $7,060(36.9)$ & $1.25(1.09-1.44)$ & $1.12(0.96-1.31)$ \\
\hline & Current daily smoker, n (\%) & $85(8.9)$ & $1,175(6.4)$ & $1.53(1.20-1.95)$ & $1.28(0.97-1.70)$ \\
\hline $\begin{array}{l}\text { Life satisfaction scale, } \\
\text { mean } \pm \text { SD }\end{array}$ & Score & $27.2 \pm 6.8$ & $28.1 \pm 6.2$ & $0.98(0.97-0.99)$ & 0.989 (0.975-0.998) \\
\hline $\begin{array}{l}\text { Social standing scale, } \\
\text { mean } \pm \text { SD }\end{array}$ & & $6.0 \pm 1.8$ & $6.3 \pm 1.8$ & $0.91(0.88-0.95)$ & $0.93(0.90-0.97)$ \\
\hline \multirow{3}{*}{$\begin{array}{l}\text { Self-rated health profile, } \\
\text { mean } \pm \text { SD }\end{array}$} & Healthy aging & $3.6 \pm 1.0$ & $3.8 \pm 0.5$ & $0.81(0.75-0.87)$ & $0.87(0.80-0.94)$ \\
\hline & Physical health & $3.6 \pm 1.0$ & $3.8 \pm 0.3$ & $0.80(0.75-0.86)$ & $0.97(0.80-0.94)$ \\
\hline & Mental health & $3.8 \pm 1.0$ & $4.0 \pm 0.5$ & $0.75(0.70-0.81)$ & $0.82(0.75-0.89)$ \\
\hline
\end{tabular}

Abbreviations: $\mathrm{Cl}$ = confidence interval; $\mathrm{OR}$ = odds ratio; $\mathrm{pRBD}$ = possible REM sleep behavior disorder.

these, 14 self-reported dementia and 44 self-reported PD/ parkinsonism, 1,386 screened positive for apnea, and 1,529 had young-onset dream enactment. This left 958 (4.9\% of the remaining 19,584 participants; $3.2 \%$ of the 30,097 -person cohort) considered as having pRBD after the removal of potential false RBD mimics (table 1).

\section{Simple multivariable analysis (age and sex adjusted only) Sociodemographic variables}

The mean age was similar between participants with pRBD and controls ( 63.0 vs 63.5 years, $\mathrm{OR}_{\mathrm{adj}} 0.994,95 \%$ confidence interval $[\mathrm{CI}] 0.988-1.001) ; 58.9 \%$ with pRBD were male vs $42.3 \%$ without $\mathrm{pRBD}\left(\mathrm{OR}_{\mathrm{adj}} .1 .97,95 \% \mathrm{CI} 1.72-2.25\right)$. There was no evidence of an association with ethnicity (white vs nonwhite $\mathrm{OR}_{\text {adj. }}$ 0.94, 95\% CI 0.70-1.29), but statistical power was limited because $95 \%$ of the cohort were white.
Those with $\mathrm{pRBD}$ were more likely to be married or in a common-law relationship $\left(\mathrm{OR}_{\text {adj. }} 1.64,95 \% \mathrm{CI} 1.22-2.17\right)$ or widowed ( $\left.\mathrm{OR}_{\text {adj. }} 1.47,95 \% \mathrm{CI} 1.02-2.12\right)$.

Those with pRBD had slightly less education (estimated mean 13.2 vs 13.6 years, $\mathrm{OR}_{\text {adj }} 0.94,95 \%$ CI $0.92-0.96$ ). They also had lower income than those without pRBD. Moreover, participants with pRBD reported having retired at a slightly younger age $\left(57.5\right.$ vs 58.6 years, $\mathrm{OR}_{\mathrm{adj}} .0 .98,95 \% \mathrm{CI}$ 0.96-0.99) and were more likely to report that retirement was due to health issues $\left(28.9 \%\right.$ vs $22.0 \%, \mathrm{OR}_{\mathrm{adj}} 1.22,95 \% \mathrm{CI}$ $1.02-1.46)$. Although veterans did not have clearly higher occurrence of pRBD (OR 1.22, 95\% CI 0.99-1.57), there was a modest relationship between self-reported years of military service and $\mathrm{pRBD}$ among veterans $\left(12.1\right.$ vs 8.9 years, $\mathrm{OR}_{\mathrm{adj}}$ 1.03, 95\% CI 1.01-1.05). 
Table 3 Mental illness $(n=19,584)$

\begin{tabular}{llllll}
\hline & & $\begin{array}{l}\text { PRBD+ } \\
\mathbf{( n = 9 5 8 )}\end{array}$ & $\begin{array}{l}\text { pRBD- } \\
\mathbf{( n = 1 8 , 6 2 6 )}\end{array}$ & $\begin{array}{l}\text { OR (95\% Cl), age/sex } \\
\text { adjusted only }\end{array}$ & $\begin{array}{c}\text { OR (95\% CI), } \\
\text { multivariate model }\end{array}$ \\
\hline Antidepressant treatment, $\mathbf{n}(\%)$ & Yes & $128(13.4)$ & $1,149(6.2)$ & $2.71(2.22-3.31)$ & $2.77(2.23-3.45)$ \\
\hline Psychological distress & K10 score, mean \pm SD & $15.2 \pm 5.3$ & $13.9 \pm 1.9$ & $1.07(1.05-1.08)$ & $1.07(1.05-1.08)$ \\
\cline { 2 - 6 } & Positive, n (\%) & $87(10.9)$ & $1,109(6.6)$ & $1.58(1.43-1.75)$ & $1.61(1.44-1.80)$ \\
\hline Mental illness, $\mathbf{n}(\%)$ & Positive & $334(34.9)$ & $4,086(21.9)$ & $2.17(1.89-2.50)$ & $2.13(1.82-2.48)$ \\
\cline { 2 - 6 } & Mood disorder & $226(23.7)$ & $2,682(14.5)$ & $2.08(1.77-2.43)$ & $2.09(1.75-2.49)$ \\
\cline { 2 - 6 } & Anxiety disorder & $132(13.8)$ & $1,355(7.3)$ & $2.24(1.85-2.72)$ & $2.18(1.75-2.70)$ \\
\cline { 2 - 6 } & Depressive disorder & $197(20.7)$ & $2,569(13.9)$ & $1.84(1.56-2.17)$ & $1.84(1.53-2.21)$ \\
\cline { 2 - 6 } & PTSD & $100(10.5)$ & $737(3.98)$ & $3.19(2.55-3.99)$ & $2.68(1.97-3.65)$ \\
\hline
\end{tabular}

Abbreviations: $\mathrm{Cl}$ = confidence interval; K10 = Kessler Psychological Distress Scale; OR = odds ratio; pRBD = possible REM sleep behavior disorder; PTSD = posttraumatic stress disorder.

\section{Activity and self-rated health}

Participants with pRBD were less likely to walk $>7 \mathrm{~h} / \mathrm{wk}$ $\left(\mathrm{OR}_{\text {adj }} 0.78,95 \% \mathrm{CI} 0.62-0.98\right)$, although the average time spent walking did not differ between groups ( $4.2 \pm 4.3$ vs $4.7 \pm$ 4.7 hours) (table 2). The frequency of participation in either social activities or social sport did not differ between those with $\mathrm{pRBD}$ and controls. Those with $\mathrm{pRBD}$ were slightly less satisfied about their lives (life satisfaction score 27.2 vs 28.1, $\mathrm{OR}_{\text {adj }} 0.98,95 \%$ CI $0.97-0.99$ ) and were more likely to rate their social standing in community as lower (mean social standing score 6.0 vs $6.3, \mathrm{OR}_{\text {adj }} 0.91,95 \%$ CI $0.88-0.95$ ). Those with pRBD self-rated as having less healthy aging (score 3.6 vs $3.8, \mathrm{OR}_{\mathrm{adj}} 0.81,95 \%$ CI $0.75-0.87$ ), physical health ( 3.6 vs $3.8, \mathrm{OR}_{\mathrm{adj}} 0.80,95 \% \mathrm{CI} 0.75-0.86$ ), and mental health (3.8 vs $4.0, \mathrm{OR}_{\text {adj }} 0.75,95 \%$ CI $\left.0.70-0.81\right)$.

\section{Alcohol use and smoking}

Participants with pRBD were more likely to drink more (100 vs $\left.70 \mathrm{~g} / \mathrm{wk}, \mathrm{OR}_{\mathrm{adj}} 1.10,95 \% \mathrm{CI} 1.03-1.17\right)$ and to be a moderate to heavy drinker ( $18.9 \%$ vs $14.3 \%, \mathrm{OR}_{\text {adj }} 1.32$, 95\% CI 1.12-1.56). Participants with pRBD were also more likely to be current smokers $\left(8.9 \%\right.$ vs $6.4 \%, \mathrm{OR}_{\mathrm{adj}} 1.61,95 \%$ CI $1.27-2.04)$ and past smokers ( $42.7 \%$ vs $36.9 \%$, $\mathrm{OR}_{\text {adj }} 1.29$, 95\% CI 1.12-1.47). The average cigarette pack-year smoking dose was slightly greater in the pRBD group ( 8 vs $6, \mathrm{OR}_{\mathrm{adj}}$ $1.01,95 \%$ CI $1.00-1.01)$.

\section{Antidepressants and mental illness}

Antidepressants were used more frequently among participants with pRBD ( $13.4 \%$ vs $6.2 \%$, OR adj $2.71,95 \%$ CI 2.22-3.31) (table 3). Participants with pRBD scored higher on the Kessler Psychological Distress Scale (15.2 \pm 5.33 vs $\left.13.9 \pm 1.86, \mathrm{OR}_{\mathrm{adj}} 1.07,95 \% \mathrm{CI} 1.05-1.08\right)$ and were more likely to report at least moderate psychological distress (10.9\% vs $6.6 \%, \mathrm{OR}_{\mathrm{adj}} 1.58,95 \%$ CI $\left.1.43-1.75\right)$. In addition, participants with $\mathrm{pRBD}$ more often had a diagnosis of mental illness $\left(34.9 \%\right.$ vs $21.9 \%, \mathrm{OR}_{\mathrm{adj}} 1.91,95 \%$ CI $\left.1.66-2.19\right)$, including a higher prevalence of physician-diagnosed anxiety (13.8\% vs $\left.7.3 \%, \mathrm{OR}_{\mathrm{adj}} 2.24,95 \% \mathrm{CI} 1.85-2.72\right)$ and depressive disorder $\left(20.7 \%\right.$ vs $13.9 \%, \mathrm{OR}_{\mathrm{adj}} 1.84,95 \% \mathrm{CI}$ 1.56-2.17). The rate of positive screening of PTSD was higher among those with pRBD $\left(10.5 \%\right.$ vs $4.0 \%, \mathrm{OR}_{\mathrm{adj}} 3.19$, 95\% CI 2.55-3.99).

\section{Full multivariable analysis}

With the multivariable logistic regression model (i.e., with all 10 variables, as listed in Methods), the associations with male sex $\left(\mathrm{OR}_{\bmod } 2.14,95 \% \mathrm{CI} 1.84-2.50\right)$ and with relationship status $\left(\mathrm{OR}_{\text {mod }} 1.77,95 \%\right.$ CI 1.45-2.16) remained. Socioeconomically, lower education level still remained a risk factor of $\mathrm{pRBD}$ in the multivariate model, but not income level, employment status, life satisfaction, or self-rated social standing. Retirement age and having reporting retirement due to health issues remained significantly associated with $\mathrm{pRBD}$, as were the amount of alcohol consumed weekly and moderate to heavy drinking $\left(\mathrm{OR}_{\bmod } 1.09,95 \% \mathrm{CI} 1.02-1.16\right.$; $\mathrm{OR}_{\text {mod }} 1.25$, 95\% CI 1.03-1.50). The average scores of life satisfaction and self-rated social standing still remained lower in $\mathrm{pRBD}$. Overall, mental illness remained highly prevalent in participants with $\mathrm{pRBD}$ compared to controls.

\section{Sensitivity analyses}

Because RLS and periodic leg movements during sleep might be confused with dream enactment, we performed a sensitivity analysis omitting any RLS screen-positive participants from the pRBD group. Of the 16,552 remaining, 756 (4.6\%) screened positive for $\mathrm{pRBD}$ (representing $2.5 \%$ of the entire population before exclusions). No substantial change in results was observed (tables e-1-e-4 available from Dryad, doi.org/10.5061/dryad.0k27q21).

To further explore pRBD in the absence of mental illness, we also removed all participants reporting anxiety, depression, high psychological stress, or PTSD. Of the 13,416 remaining 
Table 4 Sensitivity analysis after exclusion of mental illness and RLS $(n=11,609)$

\begin{tabular}{|c|c|c|c|c|c|}
\hline & & $\begin{array}{l}\text { RBD+ } \\
(n=444)\end{array}$ & $\begin{array}{l}\text { RBD- } \\
(n=11,165)\end{array}$ & Unadjusted OR & $\begin{array}{l}\text { OR }(95 \% \mathrm{CI}) \text {, age/sex } \\
\text { adjusted only }\end{array}$ \\
\hline \multirow[t]{5}{*}{ Age } & Mean \pm SD, y & $64.1 \pm 10.7$ & $63.7 \pm 10.6$ & $1.00(0.99-1.01)$ & $1.00(0.99-1.01)$ \\
\hline & $45-54$ y, n (\%) & 105 (23.6) & $2,730(24.5)$ & - & - \\
\hline & $55-64$ y, n (\%) & $132(29.7)$ & 3,393 (30.4) & $1.01(0.78-1.31)$ & $1.01(0.78-1.31)$ \\
\hline & $65-74$ y, n (\%) & $105(23.6)$ & $2,773(24.8)$ & $0.98(0.75-1.30)$ & $0.95(0.72-1.25)$ \\
\hline & $75-85$ y, n (\%) & $102(23.0)$ & $2,269(20.3)$ & $1.17(0.89-1.54)$ & $1.13(0.85-1.49)$ \\
\hline Sex, n (\%) & Male & $301(67.8)$ & $5,464(48.9)$ & $2.20(1.79-2.69)$ & $2.20(1.79-2.69)$ \\
\hline Ethnicity, n (\%) & White & $421(99.8)$ & $\begin{array}{l}10,637 \\
(99.0)\end{array}$ & $0.86(0.55-1.35)$ & $0.90(0.58-1.42)$ \\
\hline \multirow[t]{4}{*}{ Marital status, $\mathbf{n}(\%)$} & Single/never married & $18(4.5)$ & $811(7.8)$ & - & - \\
\hline & Married/common-law & $326(80.7)$ & $7,287(70.0)$ & $2.02(1.25-3.26)$ & $1.89(1.17-3.06)$ \\
\hline & Widowed & $36(8.9)$ & $1,069(10.3)$ & $1.52(0.86-2.69)$ & $1.66(0.92-3.00)$ \\
\hline & Divorced/separated & $24(5.9)$ & $1,238(11.9)$ & $0.87(0.47-1.62)$ & $1.23(0.72-2.10)$ \\
\hline \multirow[t]{4}{*}{ Education level } & Imputed years of education & $13.5 \pm 2.7$ & $13.8 \pm 2.5$ & $0.965(0.932-1.001)$ & $0.957(0.923-0.993)$ \\
\hline & BS and above, $\mathrm{n}(\%)$ & $208(46.9)$ & $5,528(49.6)$ & - & - \\
\hline & Secondary school, n (\%) & $207(46.6)$ & $5,099(45.7)$ & $1.48(0.99-2.20)$ & $1.57(1.04-2.36)$ \\
\hline & Primary/middle school, n (\%) & $29(6.5)$ & $522(4.7)$ & $1.08(0.89-1.31)$ & $1.16(0.96-1.42)$ \\
\hline \multirow[t]{4}{*}{ Annual income level, n (\%) } & $<\$ 20,000$ & 49 (11.5) & $1,286(12.2)$ & - & - \\
\hline & $\$ 20,000-\$ 49,000$ & $147(34.6)$ & $3,781(35.9)$ & $1.02(0.73-1.42)$ & $0.89(0.64-1.24)$ \\
\hline & $\$ 50,000-\$ 99,000$ & $160(37.7)$ & $3,808(36.1)$ & $1.10(0.80-1.53)$ & $0.85(0.61-1.20)$ \\
\hline & $>\$ 100,000$ & $69(16.2)$ & $1,671(15.8)$ & $1.08(0.75-1.57)$ & $0.77(0.52-1.13)$ \\
\hline \multirow[t]{4}{*}{ Employment status } & Employed, n (\%) & $180(40.6)$ & $4,763(42.8)$ & - & - \\
\hline & Retired, n (\%) & $263(59.4)$ & $6,363(57.2)$ & $1.09(0.90-1.33)$ & $1.11(0.85-1.45)$ \\
\hline & Retirement age, y & $57.5 \pm 6.8$ & $58.6 \pm 6.6$ & $1.00(0.98-1.02)$ & $0.99(0.97-1.01)$ \\
\hline & Health-related retirement, $\mathrm{n}(\%)$ & $44(16.3)$ & $1,049(16.1)$ & $1.01(0.73-1.41)$ & $1.04(0.75-1.45)$ \\
\hline \multirow[t]{2}{*}{ Military service } & Yes, n (\%) & $54(12.2)$ & $1,037(9.3)$ & $1.35(1.01-1.81)$ & $1.01(0.75-1.36)$ \\
\hline & Years of service & $15.6 \pm 13.7$ & $9.6 \pm 11.5$ & $1.04(1.02-1.06)$ & $1.04(1.02-1.06)$ \\
\hline $\begin{array}{l}\text { Satisfaction with life } \\
\text { score, mean } \pm \text { SD }\end{array}$ & Score & $29.2 \pm 5.3$ & $29.6 \pm 5.1$ & $0.99(0.97-1.01)$ & $0.99(0.97-1.01)$ \\
\hline $\begin{array}{l}\text { Social standing, } \\
\text { mean } \pm \text { SD }\end{array}$ & & $6.3 \pm 1.6$ & $6.4 \pm 1.8$ & $0.97(0.92-1.03)$ & $0.97(0.92-1.02)$ \\
\hline \multirow{2}{*}{$\begin{array}{l}\text { Frequency of social } \\
\text { activity, mean } \pm \text { SD }\end{array}$} & Social sport & $64.2 \pm 102.5$ & $68.1 \pm 13.1$ & $1.000(0.999-1.000)$ & $1.000(0.999-1.001)$ \\
\hline & Social activity & $26.8 \pm 26.7$ & $28.1 \pm 4.0$ & $0.998(0.994-1.002)$ & 0.999 (0.995-1.003) \\
\hline \multirow[t]{4}{*}{ Walk, mean \pm SD } & Hours/day & $4.2 \pm 4.3$ & $4.7 \pm 0.7$ & $0.97(0.75-0.98)$ & $0.87(0.76-0.96)$ \\
\hline & 0 h/wk, n (\%) & $60(14.7)$ & $1,387(13.1)$ & - & - \\
\hline & $<7$ h/wk, n (\%) & $269(65.8)$ & $6,515(61.7)$ & $0.95(0.72-1.27)$ & $0.97(0.73-1.29)$ \\
\hline & $\geq 7$ h/wk, n (\%) & $80(19.6)$ & $2,651(25.1)$ & $0.70(0.50-0.98)$ & $0.70(0.50-0.99)$ \\
\hline \multirow[t]{3}{*}{ Drinking } & No drinks last year, n (\%) & $34(7.9)$ & $1,097(10.1)$ & - & - \\
\hline & Occasional drinker, n (\%) & $38(8.8)$ & $1,244(11.4)$ & $1.36(0.95-1.95)$ & $1.33(0.93-1.91)$ \\
\hline & Regular drinker, n (\%) & $361(83.4)$ & $8,539(78.5)$ & $0.99(0.62-1.58)$ & $1.09(0.68-1.74)$ \\
\hline
\end{tabular}


Table 4 Sensitivity analysis after exclusion of mental illness and RLS $(n=11,609)$ (continued)

\begin{tabular}{|c|c|c|c|c|c|}
\hline & & $\begin{array}{l}\text { RBD+ } \\
(n=444)\end{array}$ & $\begin{array}{l}\text { RBD- } \\
(n=11,165)\end{array}$ & Unadjusted OR & $\begin{array}{l}\text { OR }(95 \% \mathrm{Cl}) \text {, age/sex } \\
\text { adjusted only }\end{array}$ \\
\hline & $100 \mathrm{~g}$ alcohol/wk & $1.0 \pm 2.1$ & $0.7 \pm 1.0$ & $1.16(1.10-1.24)$ & $1.12(1.05-1.21)$ \\
\hline & Binge drinking frequency & $1.5 \pm 4.9$ & $1.0 \pm 3.7$ & $1.03(1.10-1.05)$ & $1.02(1.00-1.05)$ \\
\hline & Moderate to heavy drinker, n (\%) & $84(18.9)$ & $1,630(14.6)$ & $1.37(1.07-1.74)$ & $1.47(1.15-1.88)$ \\
\hline \multirow[t]{5}{*}{ Smoking } & Cigarette pack-year all & $7.5 \pm 14.6$ & $5.6 \pm 11.6$ & $1.011(1.004-1.017)$ & $1.007(1.001-1.014)$ \\
\hline & Never daily smoking, $\%$ & $241(54.4)$ & $6,432(57.9)$ & - & - \\
\hline & $\begin{array}{l}\text { Ever smoking (reference to never } \\
\text { daily smoker), } \mathrm{n}(\%)\end{array}$ & $202(45.6)$ & $4,677(42.1)$ & $1.07(0.87-1.32)$ & $1.02(0.83-1.26)$ \\
\hline & Past daily smoker, n (\%) & $172(38.8)$ & $4,119(37.1)$ & $1.11(0.91-1.36)$ & $1.05(0.86-1.28)$ \\
\hline & Current daily smoker, $\mathrm{n}(\%)$ & $30(6.8)$ & $558(5.0)$ & $1.44(0.97-2.13)$ & $1.36(0.92-2.01)$ \\
\hline \multirow{3}{*}{$\begin{array}{l}\text { Self-rated health profile, } \\
\text { mean } \pm \text { SD }\end{array}$} & Healthy aging & $3.8 \pm 0.9$ & $3.9 \pm 0.4$ & $0.85(0.76-0.96)$ & $0.87(0.77-0.97)$ \\
\hline & Physical health & $3.9 \pm 0.9$ & $4.0 \pm 0.4$ & $0.89(0.80-1.00)$ & $0.92(0.82-1.03)$ \\
\hline & Mental health & $4.1 \pm 0.8$ & $4.2 \pm 0.4$ & $0.85(0.75-0.96)$ & $0.85(0.75-0.96)$ \\
\hline \multirow[t]{2}{*}{ Psychological distress } & K10 score & $12.9 \pm 2.8$ & $12.6 \pm 1.3$ & $1.04(1.01-1.08)$ & $1.05(1.01-1.08)$ \\
\hline & Mild, \% & $14(3.5)$ & 303 (2.9) & $1.20(0.69-2.07)$ & $1.22(0.71-2.11)$ \\
\hline
\end{tabular}

Abbreviations: $\mathrm{Cl}$ = confidence interval; $\mathrm{K} 10$ = Kessler Psychological Distress Scale; OR = odds ratio; pRBD = possible REM sleep behavior disorder; RLS = restless leg syndrome.

participants, 543 (4.0\%) screened positive for pRBD (representing $1.8 \%$ of the entire population before exclusions). Results of risk factors were generally similar to the 2 primary multivariable analyses (table e-2 available from Dryad, doi. org/10.5061/dryad.0k27q21).

Finally, to confirm our findings in absence of potential misclassification bias caused by both RLS symptoms and mental illness (including use of antidepressant), we performed an additional highly restrictive sensitivity analysis excluding any participants with pRBD endorsing either RLS or any selfreported mental illness. Of the 11,609 participants remaining, 390 (3.3\%) had pRBD (1.3\% of the total population). Risk factor results were similar to those of the regression model (table 4). It is worth noting that participants with $\mathrm{pRBD}$ were still more likely to have risky drinking habits and higher psychological distress level $(12.9 \pm 2.8$ vs $12.6 \pm 1.3)$ than controls.

\section{Discussion}

In this 30,097-participant nationwide study, we found that male sex, low education, heavy drinking, smoking, antidepressant use, and numerous indexes of mental health are linked with $\mathrm{pRBD}$.

This is a large population-based study examining risk factors for pRBD. Two previous large cross-sectional studies were conducted in Tangshan $(\mathrm{n}=12,784)$ and Shanghai $(\mathrm{n}=$ 3,635), China, ${ }^{8,21}$ that examined pRBD in selected populations. The Tangshan study found that age, male sex, marital status, low socioeconomic status, and coal mining were associated with $\mathrm{pRBD}$. Participants with $\mathrm{pRBD}$ were also more likely to smoke and to drink alcohol and coffee and were less active. The Shanghai report also found an association between risky drinking and pRBD. The Shanghai study found that those with pRBD were more likely to be single and found no difference in risk between sexes. This may be due to the nature of the cohort (67\% female participants) and the low specificity of the screening questionnaire (the RBD Screening Questionnaire was used, which includes some questions unrelated to dream enactment ${ }^{22}$ ). Three other studies were conducted with PSG-confirmed RBD., ${ }^{9,23}$ The largest, conducted by the RBD Study Group, found that RBD was more common in those with lower education, farmers, welders, and those exposed to pesticide; of note, there was no lowering of risk with caffeine and smoking (which are known to be associated with lower PD risk). A follow-up study from this group found that neither caffeine nor smoking was associated with more rapid conversion to defined PD or DLB; however, pesticide use was associated with a lower phenoconversion, and family history of dementia was associated with a higher conversion risk. ${ }^{24} \mathrm{~A}$ second PSG-proven RBD study also found that participants were more likely to be smokers, with a mild association with lower alcohol use and no relationship with caffeine use. ${ }^{23}$ Finally, a recent report from the Lausanne sleep registry again found that RBD participants were more likely to be smokers and had more antidepressant and antipsychotic use. $^{2}$ 
The strongest relationships seen in our study were between measures of mental illness and $\mathrm{pRBD}$. This same relationship has been seen in several studies, including cohorts of PSGproven RBD and RBD in association with $\mathrm{PD} .{ }^{23,25-27}$ There are several possible explanations for this finding. One is that those with mental illness may tend to endorse multiple symptoms, including multiple sleep symptoms, as part of their illness. ${ }^{28-30}$ Another is that RBD itself can lead to psychosocial distress via disruption in sleep patterns or bed partner relationships (this possibility does not accord with our clinical experience in RBD; sleep variables on PSG are generally otherwise preserved in $\mathrm{RBD},{ }^{31}$ and patients themselves often express little concern about their dream enactment). Another more compelling hypothesis is that antidepressants are well known to trigger RBD, so we may be detecting antidepressant-triggered $\mathrm{RBD} .^{27,32}$ Note, however, that the relationship with mental illness persisted even after adjustment for antidepressant use $\left(\mathrm{OR}_{\mathrm{adj}} 1.78,95 \% \mathrm{CI} 1.49-2.14\right)$. Another plausible explanation is that given that depression and anxiety, like RBD, are well-known risk factors for PD, ${ }^{27,33}$ some of the effect seen may be due to a common underlying cause (i.e., prodromal PD and DLB). Finally, it is possible that a subset of RBD exists in which people with preserved REM atonia mechanisms can nevertheless have dream enactment because of very high-intensity nightmares (common in PTSD) or general sleep-state disruption (as seen in narcolepsy). This would be consistent with the fact that the strongest relationship we observed was with PTSD and with previous descriptions of a trauma-associated sleep disorder, in which night terrors are common during both NREM and REM sleep. ${ }^{34,35}$ It is unclear whether this confound would explain all of our findings, however, because multicenter studies in PSG-confirmed cases also have found relationships between confirmed RBD and depression (OR 2.0) and antidepressant use (OR 2.4). ${ }^{36}$

Another unexplained finding, seen now in several studies, is the relationship between lower education/socioeconomic status and pRBD. ${ }^{8,9,37}$ With the comprehensive cohort of the CLSA, we were able to assess this controlling for other key variables, particularly mental illness. We saw that some of this relationship was attenuated by this adjustment, suggesting that socioeconomic status and mental illness may have partially explained the relationship. However, the residual relationship with education after adjustment remains unexplained. This could be due to residual confounding from unmeasured mental illness, a different unmeasured confounding variable, or a true causal relationship. Studies generally have not found a connection between level of education and PD or DLB, suggesting that underlying synucleinopathy is not the cause. A final possibility is that dream content (which is related to daily activities) may differ in those with less education, with a differing likelihood of being recognized by patients or bed partners (e.g., if one enacts dreams about occupational activity, physical activity during RBD might differ between a college professor and a construction worker $\left.{ }^{38}\right)$.
Given the strong and consistent inverse relationship between PD and smoking, ${ }^{8,9,23}$ the positive relationship seen here remains unexplained. Although ORs overlapped, it appeared that current smokers had the strongest association, followed by past smokers and then nonsmokers. It is known that smoking is correlated with alcohol use and mental illness. ${ }^{9,39}$ Here, with multivariable analysis, some of the relationship was attenuated after controlling for mental illness and alcohol use. It may be that nonsynucleinopathy causes of dream enactment (e.g., false positives) are positively associated with smoking, counterbalancing a "protective" role in synucleinopathy-mediated RBD. However, previous studies in PSG-confirmed RBD found that smoking was more common in RBD and was not associated with progression from idiopathic RBD to PD and DLB. Alternatively, it has been recognized that RBD is strongly associated with disease subtype in PD and DLB. ${ }^{40-43}$ It is possible that $\mathrm{PD}$ and DLB are epidemiologically heterogeneous; some subtypes are associated with smoking risk and others are not. Finally, given the complexity of factors that cause smoking behavior and the partial attenuation with multivariable adjustment, residual confounding related to unmeasured aspects of mental illness, impulse control, education, etc., may explain this relationship.

Some limitations of this study should be noted. First, the diagnosis of pRBD, although done with a validated questionnaire, nevertheless relied entirely on self-report. Of our sample, 3.18\% had pRBD; however, studies that use PSG (which should be considered the gold-standard studies) have found prevalences of $\approx 1 \%{ }^{2,3,44}$ This implies that even assuming high sensitivity, the majority of cases of pRBD do not actually have RBD. This problem is shared by all large-scale risk factors studies of RBD. ${ }^{8,21}$ In particular, most patients with NREM parasomnia would screen positive on the RBD1Q (1 study found that $69 \%$ of those with NREM parasomnia will screen positive on the RBD1Q ${ }^{45}$; we attenuated this somewhat by selecting out those who had onset before age 20 (the age at which the majority of NREM parasomnia starts). However, this would miss those who misattribute onset age and all those who develop NREM parasomnia late in life. Periodic leg movements in sleep (PLMS) can also be a source of screen positives for pRBD (noting that PLMS can also occur in true RBD). ${ }^{46}$ Although the fact that we saw no change in point estimates on sensitivity analysis when removing participants with RLS makes this less likely, there still could be confounding by those reporting PLMS in the absence of RLS. It is also possible that adding frequency information (i.e., eliminating those with infrequent events) or restricting to those with current symptoms only would help reduce the false-positive rate ${ }^{47}$; however, we do not have frequency information available. In general, incorrect diagnoses would result in a nondifferential misclassification bias; i.e., they would wash out differences between groups. If so, the significant relationships in our study would be generally stronger than what we observed, while some relationships would be missed. However, differential misclassification is 
also possible, such that false-positive screens could be driving some of the results (e.g., nonspecific sleep disturbance with depression, PTSD, apnea cases missed by screening questionnaires, periodic leg movements unassociated with RLS). As discussed, this may be particularly important for the relationship we observed between mental illness and pRBD; those with PTSD, depression, or anxiety may be particularly prone to dream enactment unrelated to loss of REM atonia. Second, it is likely that many participants would be unaware of having dream enactment. This is underscored by our finding that being married or in a long-term relationship was associated with RBD, a finding likely explained by differential levels of awareness. Third, although the CLSA is relatively comprehensive, many variables of interest were not measured such as quantitative information of caffeinated products and other substance consumption (dietary information has been collected, but analysis is pending). Fourth, because we were studying idiopathic RBD, we eliminated self-reported parkinsonism or PD. Overall sensitivity for self-report PD approximates $80 \%$, meaning that some patients with true PD might have been missed (note that dementia was eliminated with both self-report and cognitive examination, so this limitation would not apply for dementia).

On the other hand, the main advantages of our current study are the large sample size, the systematic population-based sampling, the capacity to adjust for diverse potential confounding variables, including mental illness, and the ability to screen out RBD mimics such as apnea, RLS, and possible NREM parasomnia.

This study has replicated findings originally seen in smaller-scale cohorts that smoking, low education, and male sex are associated with RBD. We found a previously unreported link with alcohol use. Finally, we found a strong connection between pRBD and mental illness in general, including depression, anxiety, and PTSD. Further clinical research on the comorbidity of mental illness and psychological health profile in idiopathic RBD is needed to disentangle the complex interplay of sleep and mental health. Extra attention to mental health issues in the clinical care of patients with RBD may be warranted.

\section{Author contributions}

Chun Yao: research project: conception; statistical analysis: design, execution, review, and critique; manuscript preparation: writing of the first draft, review, and critique. SeyedMohammad Fereshtehnejad: research project: conception; statistical analysis: design, review, and critique; manuscript preparation: review and critique. Mark R. Keezer: research project: conception, critique; statistical analysis: review and critique; manuscript preparation: review, and critique. Christina Wolfson: research project: organization, conception, critique; statistical analysis: review and critique; manuscript preparation: review and critique. Amélie Pelletier: research project: organization, execution; statistical analysis: review and critique; manuscript preparation: review and critique. Ronald B. Postuma: research project: conception, organization, execution, critique; statistical analysis: design, review and critique; manuscript preparation: writing of the first draft, review and critique.

\section{Acknowledgment}

This research was made possible with the data/biospecimens collected by the CLSA. The authors are grateful for all participants' contributions to the study and the opportunity provided by CLSA.

\section{Study funding}

Funding for the CLSA was provided by the Government of Canada through the Canadian Institutes of Health Research (CIHR) under grant reference LSA 9447 and the Canada Foundation for Innovation and by CIHR operating grant ACD 151284 (E.F.). This research has been conducted with the CLSA Baseline Comprehensive Dataset version 3.1 under Application No. 160601. The CLSA is led by Drs. Parminder Raina, Christina Wolfson, and Susan Kirkland. This research was funded by CIHR, the Webster Foundation, and the Fonds de la Recherche-Sante Quebec.

\section{Disclosure}

C. Yao and S. Fereshtehnejad report no disclosures relevant to the manuscript. M. Keezer reports speaker and advisory fees from Elsevier, Sunovion, Novartis, Sage Therapeutics, and UCB; unrestricted educational grants from UCB; and research grants from UCB, Eisai, and the Centre de recherche de l'Université de Montréal. C. Wolfson reports grants from the CIHR and the Multiple Sclerosis Society of Canada. A. Pelletier reports no disclosures relevant to the manuscript. $\mathrm{R}$. Postuma reports grants and personal fees from Fonds de la Recherche en Sante; grants from CIHR, The Parkinson Society of Canada, Weston-Garfield Foundation, Michael J. Fox Foundation, and Webster Foundation; and personal fees from Biotie, Roche/Prothena, Teva Neurosciences, Novartis Canada, Biogen, Boehringer Ingelheim, Theranexus, and GE HealthCare. Go to Neurology.org/N for full disclosures.

\section{Publication history}

Received by Neurology January 11, 2018. Accepted in final form October 1, 2018.

\section{References}

1. Kang SH, Yoon IY, Lee SD, Han JW, Kim TH, Kim KW. REM sleep behavior disorder in the Korean elderly population: prevalence and clinical characteristics. Sleep 2013; 36:1147-1152.

2. Haba-Rubio J, Frauscher B, Marques-Vidal P, et al. Prevalence and determinants of REM sleep behavior disorder in the general population. Sleep 2017;41:zsx197.

3. Pujol M, Pujol J, Alonso T, et al. Idiopathic REM sleep behavior disorder in the elderly Spanish community: a primary care center study with a two-stage design using videopolysomnography. Sleep Med 2017;40:116-121.

4. Li SX, Wing YK, Lam SP, et al. Validation of a new REM sleep behavior disorder questionnaire (RBDQ-HK). Sleep Med 2010;11:43-48.

5. Boeve BF, Molano JR, Ferman TJ, et al. Validation of the Mayo Sleep Questionnaire to screen for REM sleep behavior disorder in an aging and dementia cohort. Sleep Med 2011;12:445-453.

6. Frauscher B, Ehrmann L, Zamarian L, et al. Validation of the Innsbruck REM Sleep Behavior Disorder Inventory. Mov Disord 2012;27:1673-1678.

7. Postuma RB, Arnulf I, Hogl B, et al. A single-question screen for rapid eye movement sleep behavior disorder: a multicenter validation study. Mov Disord 2012;27: 913-916.

8. Wong JC, Li J, Pavlova M, et al. Risk factors for probable REM sleep behavior disorder: a community-based study. Neurology 2016;86:1306-1312. 
9. Postuma RB, Montplaisir JY, Pelletier A, et al. Environmental risk factors for REM sleep behavior disorder: a multicenter case-control study. Neurology 2012;79: 428-434.

10. Raina PS, Wolfson C, Kirkland SA, et al. The Canadian Longitudinal Study on Aging (CLSA). Can J Aging 2009;28:221-229.

11. Markov D, Jaffe F, Doghramji K. Update on parasomnias: a review for psychiatric practice. Psychiatry 2006;3:69-76.

12. Fleetham JA, Fleming JA. Parasomnias. CMAJ 2014;186:E273-E280.

13. Postuma RB, Gagnon JF, Bertrand JA, Genier Marchand D, Montplaisir JY. Parkinson risk in idiopathic REM sleep behavior disorder: preparing for neuroprotective trials. Neurology 2015;84:1104-1113.

14. Chung F, Abdullah HR, Liao P. STOP-BANG Questionnaire: a practical approach to screen for obstructive sleep apnea. Chest 2016;149:631-638.

15. Adler NE, Epel ES, Castellazzo G, Ickovics JR. Relationship of subjective and objective social status with psychological and physiological functioning: preliminary data in healthy white women. Health Psychol 2000;19:586-592.

16. Van Dam NT, Earleywine M. Validation of the Center for Epidemiologic Studies Depression Scale-Revised (CESD-R): pragmatic depression assessment in the general population. Psychiatry Res 2011;186:128-132.

17. Prins A, Bovin MJ, Smolenski DJ, et al. The Primary Care PTSD Screen for DSM-5 (PC-PTSD-5): development and evaluation within a veteran primary care sample. J Gen Intern Med 2016;31:1206-1211.

18. Kessler RC, Andrews G, Colpe LJ, et al. Short screening scales to monitor population prevalences and trends in non-specific psychological distress. Psychol Med 2002;32: 959-976.

19. Hattan E, Chalk C, Postuma RB. Is there a higher risk of restless legs syndrome in peripheral neuropathy? Neurology 2009;72:955-960.

20. Hening WA, Allen RP, Washburn M, Lesage S, Earley CJ. Validation of the Hopkins telephone diagnostic interview for restless legs syndrome. Sleep Med 2008;9: 283-289.

21. Ma JF, Qiao Y, Gao X, et al. A community-based study of risk factors for probable rapid eye movement sleep behavior disorder. Sleep Med 2017;30:71-76.

22. Stiasny-Kolster K, Mayer G, Schafer S, Moller JC, Heinzel-Gutenbrunner M, Oertel WH. The REM Sleep Behavior Disorder Screening Questionnaire: a new diagnostic instrument. Mov Disord 2007;22:2386-2393.

23. Baig F, Lawton MA, Rolinski M, et al. Personality and addictive behaviours in early Parkinson's disease and REM sleep behaviour disorder. Parkinsonism Relat Disord 2017;37:72-78.

24. Postuma RB, Iranzo A, Hogl B, et al. Risk factors for neurodegeneration in idiopathic rapid eye movement sleep behavior disorder: a multicenter study. Ann Neurol 2015; 77:830-839.

25. Rolinski M, Szewczyk-Krolikowski K, Tomlinson PR, et al. REM sleep behaviour disorder is associated with worse quality of life and other non-motor features in early Parkinson's disease. J Neurol Neurosurg Psychiatry 2014;85:560-566.

26. Wing YK, Lam SP, Li SX, et al. REM sleep behaviour disorder in Hong Kong Chinese: clinical outcome and gender comparison. J Neurol Neurosurg Psychiatry 2008;79: 1415-1416.

27. Postuma RB, Gagnon JF, Tuineaig M, et al. Antidepressants and REM sleep behavio disorder: isolated side effect or neurodegenerative signal? Sleep 2013;36:1579-1585.
28. Nutt D, Wilson S, Paterson L. Sleep disorders as core symptoms of depression. Dialogues Clin Neurosci 2008;10:329-336.

29. Horvath A, Montana X, Lanquart JP, et al. Effects of state and trait anxiety on sleep structure: a polysomnographic study in 1083 subjects. Psychiatry Res 2016;244: 279-283.

30. Baglioni C, Nanovska S, Regen W, et al. Sleep and mental disorders: a meta-analysis of polysomnographic research. Psychol Bull 2016;142:969-990.

31. Postuma RB, Gagnon JF, Pelletier A, Montplaisir JY. Insomnia and somnolence in idiopathic RBD: a prospective cohort study. NPJ Parkinsons Dis 2017;3:9.

32. Gagnon JF, Postuma RB, Montplaisir J. Update on the pharmacology of REM sleep behavior disorder. Neurology 2006;67:742-747.

33. Berg D, Postuma RB, Adler $\mathrm{CH}$, et al. MDS research criteria for prodromal Parkinson's disease. Mov Disord 2015;30:1600-1611.

34. Mysliwiec V, Brock MS, Creamer JL, O’Reilly BM, Germain A, Roth BJ. Trauma associated sleep disorder: a parasomnia induced by trauma. Sleep Med Rev 2018;37: 94-104.

35. Manni R, Ratti PL, Terzaghi M. Secondary “incidental” REM sleep behavior disorder: do we ever think of it? Sleep Med 2011;12(suppl 2):S50-S53.

36. Frauscher B, Jennum P, Ju YE, et al. Comorbidity and medication in REM sleep behavior disorder: a multicenter case-control study. Neurology 2014;82:1076-1079.

37. Wing YK, Lam SP, Tsoh JM, Mok VC. Rapid eye movement sleep behaviour disorder and psychiatry: a case-control study. Hong Kong Med J 2015;21(suppl 6):34-38.

38. Stiasny-Kolster K, Sixel-Doring F, Trenkwalder C, et al. Diagnostic value of the REM Sleep Behavior Disorder Screening Questionnaire in Parkinson's disease. Sleep Med 2015;16:186-189.

39. Ziedonis D, Hitsman B, Beckham JC, et al. Tobacco use and cessation in psychiatric disorders: National Institute of Mental Health report. Nicotine Tob Res 2008;10: $1691-1715$

40. Fereshtehnejad SM, Zeighami Y, Dagher A, Postuma RB. Clinical criteria for subtyping Parkinson's disease: biomarkers and longitudinal progression. Brain 2017;140: 1959-1976.

41. Postuma RB, Bertrand JA, Montplaisir J, et al. Rapid eye movement sleep behavior disorder and risk of dementia in Parkinson's disease: a prospective study. Mov Disord 2012;27:720-726.

42. Postuma RB, Adler CH, Dugger BN, et al. REM sleep behavior disorder and neuropathology in Parkinson's disease. Mov Disord 2015;30:1413-1417.

43. Dugger BN, Boeve BF, Murray ME, et al. Rapid eye movement sleep behavior disorder and subtypes in autopsy-confirmed dementia with Lewy bodies. Mov Disord 2012;27:72-78.

44. Baig F, Lawton M, Rolinski M, et al. Delineating nonmotor symptoms in early Parkinson's disease and first-degree relatives. Mov Disord 2015;30:1759-1766.

45. Haridi M, Weyn Banningh S, Cle M, Leu-Semenescu S, Vidailhet M, Arnulf I. Is there a common motor dysregulation in sleepwalking and REM sleep behaviour disorder? J Sleep Res 2017;26:614-622.

46. Fantini ML, Michaud M, Gosselin N, Lavigne G, Montplaisir J. Periodic leg movements in REM sleep behavior disorder and related autonomic and EEG activation. Neurology 2002;59:1889-1894.

47. Nielsen T, Svob C, Kuiken D. Dream-enacting behaviors in a normal population. Sleep 2009;32:1629-1636. 